\title{
Tanshinone IIA Improves miR-133 Expression Through MAPK ERK1/2 Pathway in Hypoxic Cardiac Myocytes
}

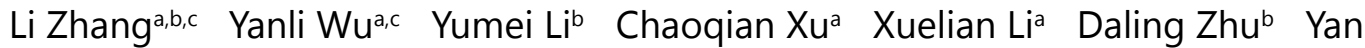 \\ Zhang $^{\text {a }}$ Shu Xing ${ }^{\text {a Haoyan Wang }}{ }^{\text {a }}$ Zhihua Zhanga Hongli Shan $^{a}$ \\ aDepartment of Pharmacology (State-Province Key Laboratories of Biomedicine- Pharmaceutics \\ of China, Key Laboratory of Cardiovascular Research, Ministry of Education), and 'Department of \\ Pharmacology, and the central lab of Harbin Medical University (Daqing campus), Daqing, Heilongjiang \\ 150081, P. R. China; 'The authors made equal contributions to this study
}

\section{Key Words}

Tanshinone IIA $・$ miR-133 $・$ ERK1/2 $・$ Hypoxia

\begin{abstract}
Tanshinone IIA is a lipid-soluble pharmacologically active compound extracted from the rhizome of Chinese herb Salvia miltiorrhiza, a well-known traditional Chinese medicine used for the treatment of cardiovascular disorders. Previous studies have identified that tanshinone IIA inhibited overexpression of miR-1 in hypoxic neonatal cardiomyocytes. This study was designed to examine the effects of tanshinone IIA on miR-133 expression under hypoxic condition. Neonatal rat cardiomyocytes were cultured in a hypoxic environment $\left(2 \% \mathrm{O}_{2}+93 \% \mathrm{~N}_{2}+5 \%\right.$ $\mathrm{CO}_{2}$ ) at $37^{\circ} \mathrm{C}$ for 24 hours. MTT, TUNEL assays, and Flow Cytometry (FCM) were performed to identify cell apoptosis. Western blot was used to examine the expression of ERK1/2 and miR133 level was quantified by Real-time PCR. Our results showed that apoptosis was induced by hypoxia. Typical apoptotic cells were seen by TUNEL assay, and FCM showed an apoptosis rate of $13.32 \%$ in hypoxic group. Apoptosis rate in hypoxic cells was reduced significantly by tanshinone IIA. In addition, the expression level of miR-133 was increased in hypoxic cells and further upregulated by tanshinone IIA. The stress-activated protein kinase MAPK ERK1/2 was activated by hypoxia and further increased with tanshinone IIA treatment. The present study demonstrated that tanshinone IIA enhanced cell resistance to hypoxic insult by upregulating miR-133 expression through activating MAPK ERK1/2 in neonatal cardiomyocytes.
\end{abstract}




\section{Introduction}

MicroRNAs (miRNAs) are a family of endogenous small, noncoding RNAs, about 22 nucleotides in length. These molecules mediate post-transcriptional gene silencing by annealing to inexactly complementary sequences in the 3'-untranslated regions of target mRNAs [1]. As the key regulating player of gene expression, miRNAs are essential for cardiac function, including conductance of electrical signals, heart muscle contraction, development and morphogenesis. MiRNAs are also involved in proliferation and apoptosis in cardiomyocytes, cardiac hypertrophy, and heart failure [2-4]. Among identified mammalian miRNAs, muscle specific miRNAs (miR-1 and miR-133) are believed to be important regulators of myogenesis [5]. Recent studies demonstrated that upregulation or downregulation of either of them might influence the pathogenesis of cardiac diseases [68]. MiR-133 was proved to control cardiac hypertrophy, regulate connective tissue growth factor, and contribute to atrial remodeling in canines $[4,7,9]$. It also played an important role in ischemic post-conditioning protection by regulating apoptosis-related genes, caspase- 9 [10].

Tanshinone IIA is a lipid-soluble monomer derivative of phenanthrenequinone extracted from the root of Salvia miltiorrhiza (Danshen). It has been shown to possess a variety of biological activities in cardiovascular system, such as antioxidant, anticoagulant, antiatherosclerosis, antiapoptosis, and antihypertrophy [11-15]. It was widely used for the treatment of cardiovascular diseases for example coronary heart diseases. Accumulated evidences suggested that tanshinone IIA reduced ischemic infarction area and improved cardiac function $[13,16]$. Furthermore, our previous work had identified that tanshinone IIA protected cardiomyocytes by inhibiting elevated miR-1 expression during myocardial infarction and hypoxia-ischemia [17]. It was further confirmed that downregulation of miR1 by tanshinone IIA was due to blocking cardiac p38 MAPK [18]. Yang et al. also reported that sodium tanshinone IIA sulfonate protected cardiomyocytes against oxidative stressmediated apoptosis through inhibiting JNK activation [15]. However, it remains unclear whether tanshinone IIA regulates miR-133 level under hypoxic condition.

ERK (extracellular signal regulated kinase), an important mitogen-activated protein kinase (MAPK) family member, regulates cell proliferation and differentiation. Activated ERK1/2 MAP Kinase translocates to the nucleus and activates transcription byphosphorylation of transcription factors such as SRF (Serum response factor) and c-fos [19]. Hypoxia activates certain signaling pathways (MAPK ERK) in heart, and induces gene expression, such as hypoxia-inducible factor 1 (HIF-1) which activates transcription of genes [20]. Hypoxia also induces apoptosis in cultured neonatal rat cardiomyocytes and cardiac fibroblasts [21, 22]. The ERK1/2 signaling pathway has been implicated as a survival signaling pathway in response to ischemia/reperfusion [23, 24]. Inhibition of ERK1/2 signaling pathway could increase $\mathrm{H}_{2} \mathrm{O}_{2}$-induced apoptosis in cultured cardiomyocytes [25]. Although studies have shown that stress or agonist-induced ERK1/2 activation has been associated with protection against apoptosis, little is known about how the ERK1/2 signaling pathway is controling cell apoptosis via miRNAs.

The present study was designed to investigate the effect of tanshinone IIA on hypoxiainduced injury and the modulation of miR-133 via MAPK ERK1/2 signaling pathway in cultured neonatal ventricular cardiomyocytes.

\section{Materials and Methods}

\section{Drugs}

Tanshinone IIA (purity 99\%) was purchased from Xian Guanyu Bio-tech Co. Ltd, China. U0126, a highly selective inhibitor of ERK1/2, was purchased from Sigma, USA. They were dissolved in dimethylsulphoxide (DMSO). The final concentration of DMSO did not exceed $0.1 \%$. 


\section{Isolation and culture of cardiomyocytes}

Primary cultures of neonatal rat cardiomyocytes were prepared from 1 to 3-day-old Wistar rats by trypsin as described previously [26, 27]. The ventricular myocardium was minced in DMEM (Dulbecco's Modified Eagle Medium, Hyclone, USA), which contains $25 \mathrm{mM}$ D-glucose and $4 \mathrm{mM}$ L-Glutamine. After each of six successive 6 min incubation, the cells were suspended in DMEM containing 10\% FBS (Fetal Bovine Serum, Hyclone, USA) and centrifuged. Pooled cells were plated onto dishes at a density of $10^{5} \mathrm{cells} \cdot \mathrm{cm}^{-2}$ and incubated at $37^{\circ} \mathrm{C}$ in a humidified air with $5 \% \mathrm{CO}_{2}$ for $72 \mathrm{~h}$, and $0.1 \mathrm{mM}$ bromodeoxyuridine (Sigma, USA) was added into the medium to deplete nonmyocytes. Before the experiment, the cells starved to serum-free DMEM for another $24 \mathrm{~h}$, and were then treated with various agents. Neonatal cardiomyocytes were cultured under a condition of $5 \% \mathrm{CO}_{2}-2 \% \mathrm{O}_{2}-93 \% \mathrm{~N}_{2}$ for $24 \mathrm{~h}$. Tanshinone IIA and U0126 were given $30 \mathrm{~min}$ preceding hypoxia expose. The image data were analyzed with Image-Pro Plus software (Media. Cybernetic, USA).

\section{MTT assay for cell viability}

Cell Proliferation Kit I [3-(4,5-dimethylthiazol-2-yl)-2,5-diphenyl tetrazolium bromide] (MTT) (Roche Molecular Biochemicals, Laval, $\mathrm{PQ}$, Canada) was used to quantify survival of cells suffering from hypoxic injury or tanshinone IIA treatment. After trypan blue test, cells were plated at 10,000 cells per $\mathrm{cm}^{2}$ in 96 well plates. After $24 \mathrm{~h}$ different treatment, $20 \mu \mathrm{L}$ of MTT solution was added to each well, and the cells were incubated for $4 \mathrm{~h}$ at $37^{\circ} \mathrm{C}$. The supernatants were removed, then the cell layer was dissolved with $150 \mu \mathrm{L}$ DMSO. The plate was placed on a shaker for $15 \mathrm{~min}$ for solubilization of crystals and then the optical density of each well was determined at $490 \mathrm{~nm}$ using a Model 680 Microplate Reader (Bio-Rad, USA).

\section{Terminal Deoxyribonucleotide Transferase-Mediated dUTP Nick End Labeling (TUNEL)}

DNA fragmentation of individual cells was detected in situ by TUNEL with the In Situ Cell Death Detection kit, (Roche Molecular Biochemicals, USA), following the manufacturer's instructions. Briefly, Dewax paraffin-embedded sections in xylene and rehydrate were carried out in graded ethanol series to water. Permeabilization of tissue with $20 \mu \mathrm{g} \cdot \mathrm{mL}^{-1}$ Proteinase $\mathrm{K}$, and TUNEL labeling were done, as detailed elsewhere [28].

\section{Flow Cytometry}

Cell apoptosis detection was performed by Flow Cytometry (FCM) analysis, using an "In Situ Cell Death Detection" kit (BD PharMingen, USA) following the manufacturer's instructions, as previously described [29]. Briefly, primary cultured cadiomyocytes were exposed to hypoxia for 24 hours with or without tanshinone IIA treatment. The cardiomycytes were collected, washed twice with ice-cold PBS $(137 \mathrm{mM} \mathrm{NaCl}, 1.47 \mathrm{mM}$ $\mathrm{KH}_{2} \mathrm{PO}_{4}, 7.81 \mathrm{mM} \mathrm{Na}_{2} \mathrm{HPO}_{4}, 2.68 \mathrm{mM} \mathrm{KCl}, \mathrm{PH} 7.40$, BSD, China), and then were suspended in $200 \mu \mathrm{L}$ of binding buffer (10× binding buffer $0.1 \mathrm{M}$ Hepes, $1.4 \mathrm{M} \mathrm{NaCl}, 25 \mathrm{mM} \mathrm{CaCl}_{2}, \mathrm{pH}$ 7.4, BD Pharmingen, USA) and $10 \mu \mathrm{L}$ of Annexin V-FITC (BD Pharmingen, USA) for 15 minutes in the dark. Thereafter, $300 \mu \mathrm{L}$ of binding buffer and $5 \mu \mathrm{L}$ of propidium iodide (BD Pharmingen, USA) were added to each sample. Finally, the cells were analyzed using BD Flow Cytometry (BD FACS Canto II, USA) with Cell Quest software (BD Biosciences, USA).

\section{Quantitative Real-time PCR}

Total RNA samples were extracted using Trizol (Invitrogen, USA) from cultural myocytes. miR-133 level was quantified by the mirVana qRT-PCR (quantitative real-time PCR) miRNA Detection Kit (Ambion, USA) in conjunction with real-time PCR with SYBR Green I (Applied Biosystems, USA), as described in detail [4, 8]. Reverse transcription primers for miR-133a was: 5'- GTCGTATCCAGTGCGTGTCGTGGAGTCGGCAATTGCACT GGATACGACCAGCTG-3'. The following primers were used for PCR detection: 5'- GGGTTTGGTCCCCTTCAA3' (forward); 5'-AGTGCGTGTCGTGGAGTC-3' (reverse). U6 was used as an internal control. The relative expression of miR-133 was calculated and normalized to U6 using the comparative $\mathrm{Ct}$ method. Relative expression intensity values were calculated as $2^{-\Delta \Delta} \mathrm{Ct}$.

\section{Western Blot Analysis}

The membrane protein samples were extracted from primary cultured neonatal rat ventricular myocytes for immunoblotting analysis of ERK1/2 with the procedure as previously described [29]. The protein content was determined with BCA Protein Assay Kit (Bio-Rad, Mississauga, ON, Canada). Equal amounts of 


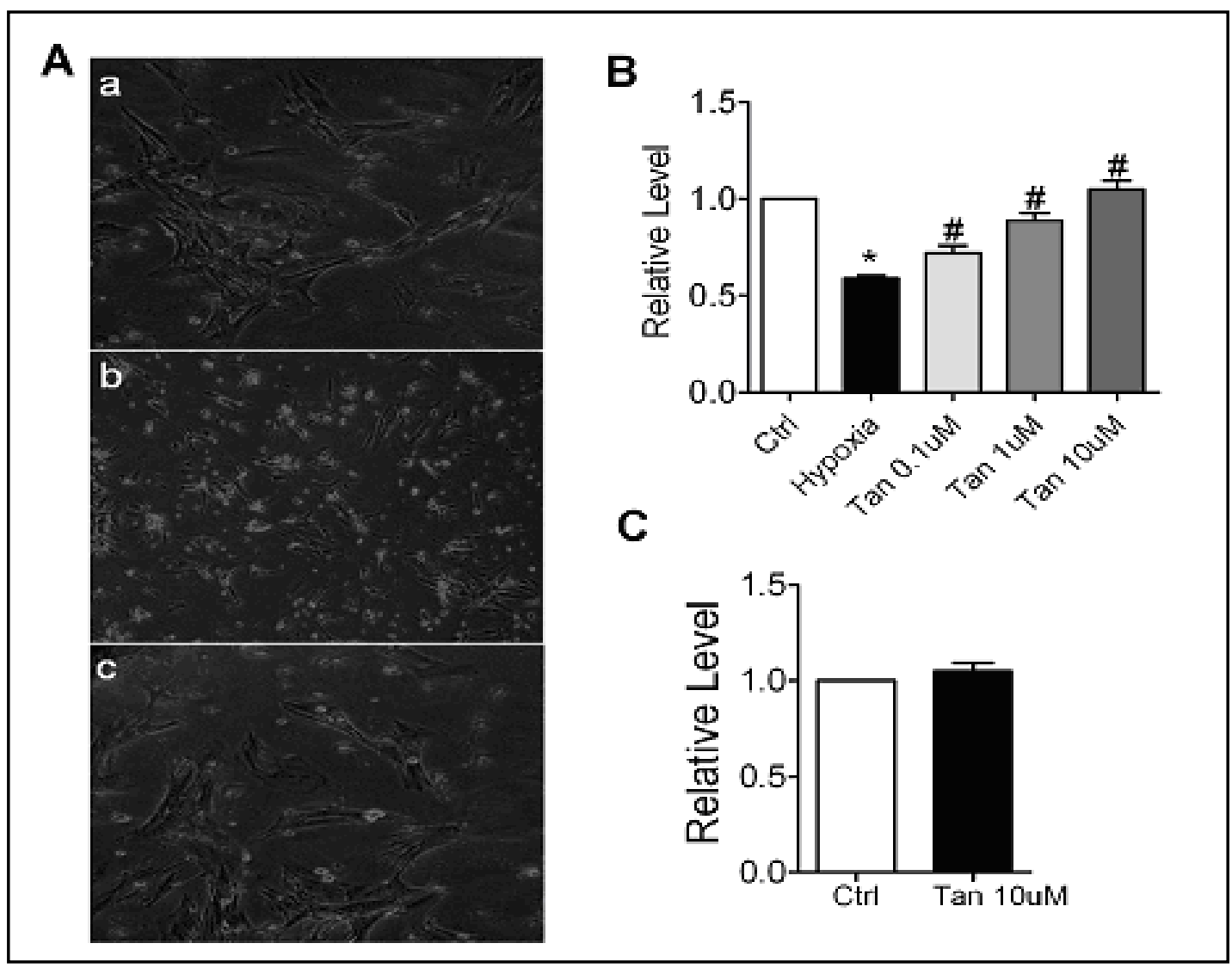

Fig. 1. Tanshinone IIA protects neonatal rat ventricular cells (NRVCs) against hypoxia. (A) Morphological manifestations of cultured neonatal rat cardiomyocytes (NRVCs) under conditions of normoxia (a), hypoxia (b), and hypoxia with tanshinone IIA treatment (Tan) (c). (B) Tanshinone IIA increased cell viability in a concentration - dependent manner. Cells were incubated under hypoxic condition. Tanshinone IIA was given 30 min preceding hypoxia exposure in different doses $(0.1,1,10 \mu \mathrm{M})$. Cell viability was determined by MTT assay. (C) Tanshinone IIA $(10 \mu \mathrm{M})$ showed no cell toxicity $(\mathrm{p}>0.05)$.

protein $(60 \mu \mathrm{g})$ were loaded on a 10\% SDS-PAGE gel. The lysates were resolved by electrophoresis $(70 \mathrm{~V}$ for $30 \mathrm{~min}$ and $100 \mathrm{~V}$ for $1.5 \mathrm{~h}$ ) and transferred onto NC membranes (nitrocellulose membrane, Bio-Rad, USA). After blocking in 5\% nonfat milk for $2 \mathrm{~h}$ at room temperature the NC membrane were treated with antipERK1/2 or t-ERK1/2 (1:200, Cell Signaling Technology, USA). Next day, the NC membrane (nitrocellulose membrane, Bio-Rad, USA) was washed in PBS for three times (15 min/each) and incubated for $1.5 \mathrm{~h}$ with the fluorescence-conjugated anti-rabbit IgG (1:4000) in the blocking buffer. GAPDH (glyceraldehyde-3phosphate) was used as an internal control for equal input of protein samples. Both primary and secondary antibodies were purchased from Santa Cruz Biotechnology (Santa Cruz, CA, USA). Western blot bands were quantified using Odyssey v1.2 software (Infrared Imaging System LI-COR Biosciences) by measuring the band intensity (Area×OD, Optical Density) for each group and normalized by GAPDH. The final results are expressed as fold changes by normalizing the data to the control values.

\section{Data Analysis}

All data are presented as mean \pm SEM. There are more than two groups of continuous variables in this study, so one-way analysis of variance ANOVA (Analysis Of Variance) followed by Bonferroni or Dunnett's post-hoc test was used for multiple comparisons. A two-tailed value of $p<0.05$ is considered statistically significant difference. Data are analyzed using the GraphPad Prism 5.0 and SPSS 14.0. 


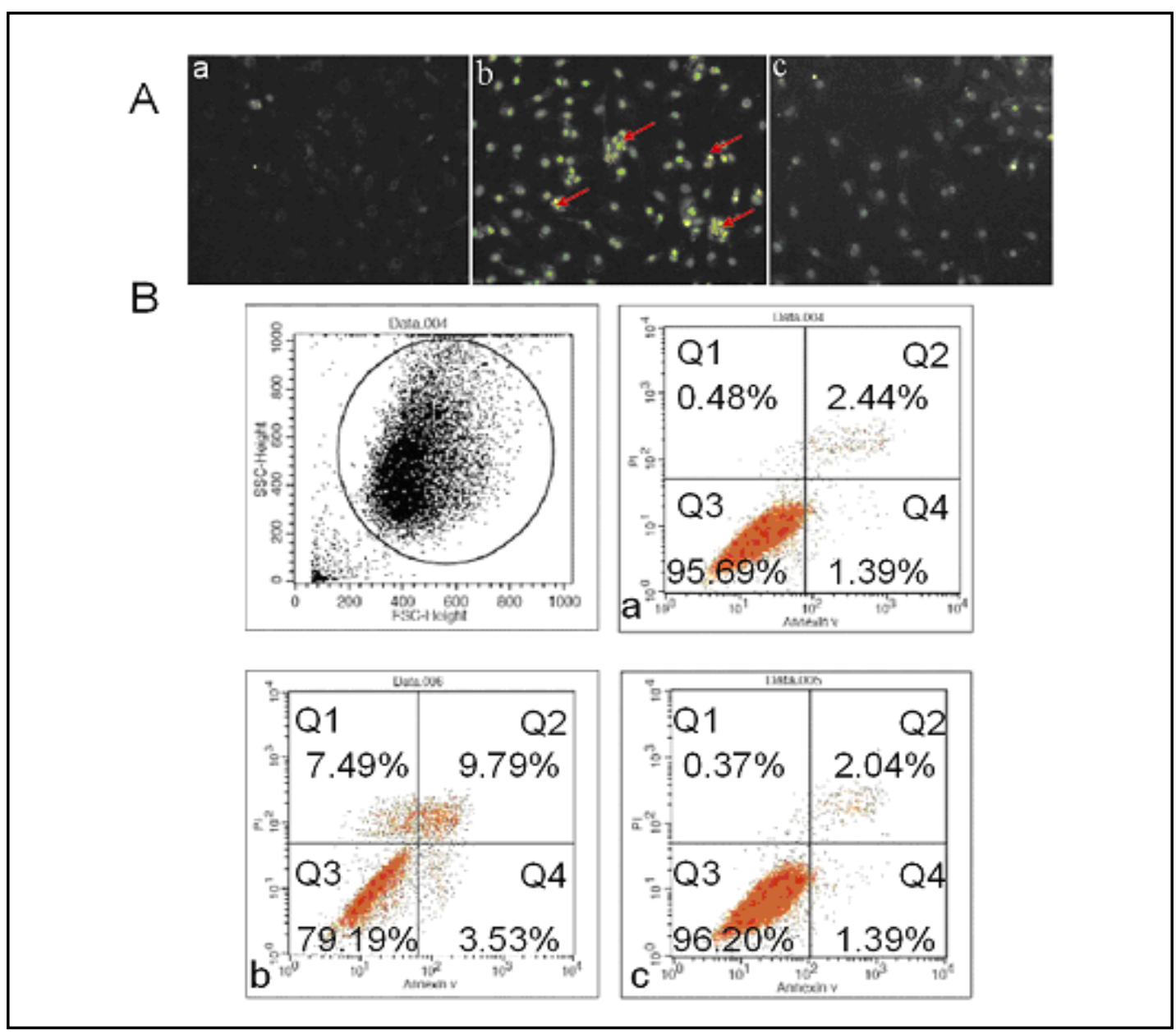

Fig. 2. Tanshinone IIA significantly decreases cell apoptosis induced by hypoxia in NRVCs. (A) Cell apoptosis was assessed by TUNEL assay. Representative TUNEL staining of NRVCs shows no cell apoptosis (TUNELnegative) in normoxia group (a); notable cell apoptosis (TUNEL-positive, arrows) in hypoxia group (b); and dramatically decreased apoptotic cell number in hypoxia with tanshinone IIA treatment group (c). (B) Cell apoptosis was assessed by Flow Cytometry. The percentage of cells in each quadrant is indicated in the quadrant (n=3). Q1 represents necrotic cells, Q2 represents late apoptotic cells, Q3 represents viable cell, and Q4 represents early apoptotic cells. The experiment was performed in triplicate for three independent times.

\section{Results}

Tanshinone IIA increases cell viability in hypoxic cardiomyocytes

Neonatal rat ventricular myocytes were cultured under hypoxic condition for $24 \mathrm{~h}$. Cell size was decreased obviously in hypoxia, but it was reversed with tanshinone IIA treatment as shown in Fig. 1A. Moreover, the reduced cell viability caused by hypoxia was increased with tanshinone IIA treatment in a concentration-dependent way (Fig. 1B). Even the maximal dose of tanshinone IIA $(10 \mu \mathrm{M})$ showed no cell toxicity (Fig. 1C).

Tanshinone IIA protects cell against apoptosis

TUNEL and Flow Cytometry were performed to examine apoptosis in hypoxic cardiomyocytes with or without tanshinone IIA treatment for $24 \mathrm{~h}$. TUNEL-positive cell number induced by hypoxia was significantly reduced when hypoxic cells were treated with tanshinone IIA (Fig. 2A). There was a significant increase in cell apoptosis caused by hypoxic injury. Tanshinone IIA markedly decreased the apoptosis rate induced by hypoxia (Fig. 2B). 
Fig. 3. Tanshinone IIA enhances miR-133 level after hypoxia. The effect of Tanshinone IIA on miR-133 level was examined using Real-time PCR. Results are expressed as fold changes compared with vehicletreated samples which was set as 1 . Data are plotted as the mean \pm S.E.M. ${ }^{*} \mathrm{p}<0.05$ vs. control; $\# \mathrm{p}<0.05$ vs. hypoxia, $\mathrm{n}=5$.

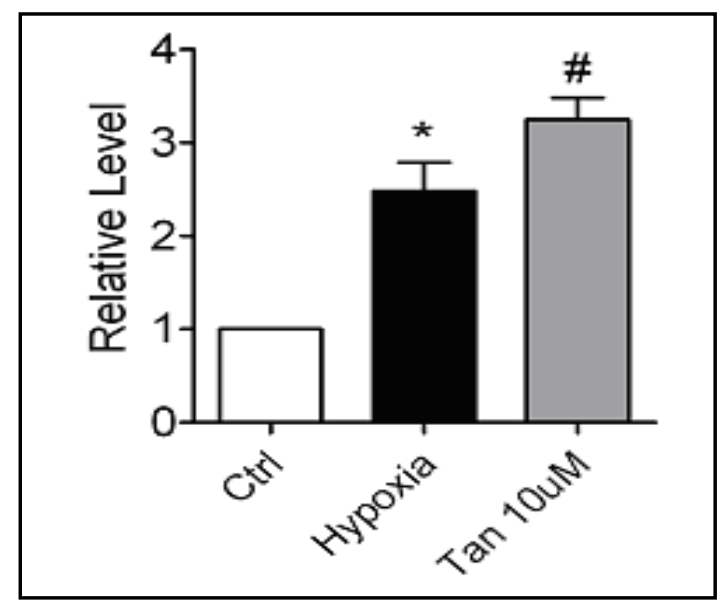

Fig. 4. Effect of Tanshinone IIA on phosphorylationERK (p-ERK) in hypoxic NRVCs. NRVCs were pretreated with Tanshinone IIA $(10 \mu \mathrm{M})$ for 30 minutes and subjected to hypoxia $\left(2 \% \mathrm{O}_{2}\right)$ for $24 \mathrm{~h}$, and the phosphorylation levels of ERK1/2 were determined by Western blot analysis. Total ERK (t-ERK) protein values were measured in parallel. GAPDH served as a loading control. ${ }^{*} \mathrm{P}<0.05$ vs control, \#P $<0.05$ vs hypoxia, $\mathrm{n}=5$.

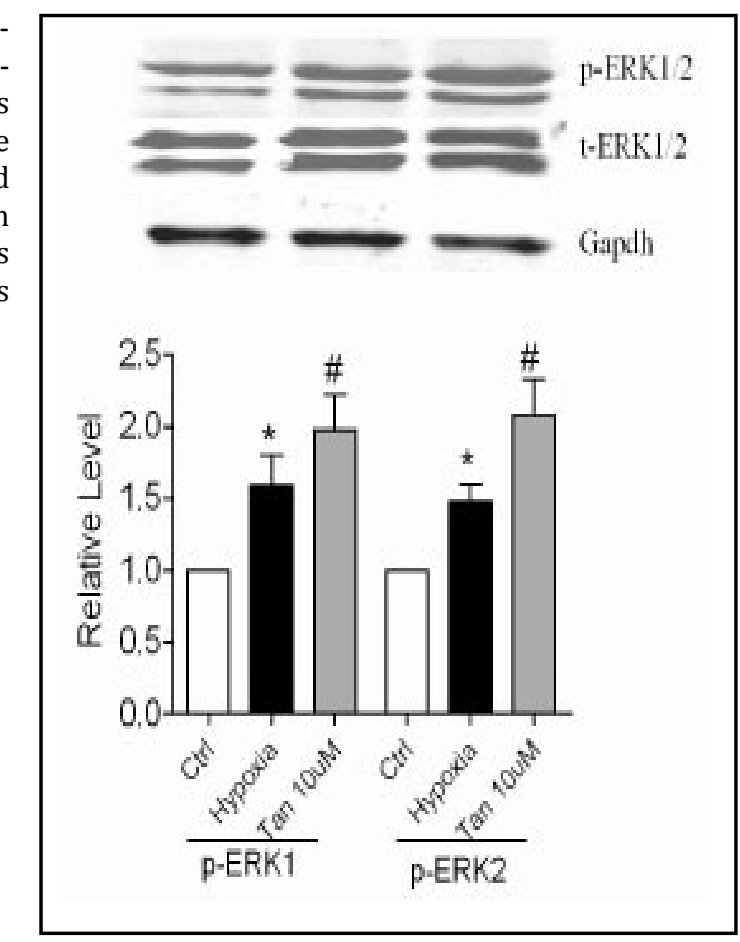

Cell apoptosis rate was raised to $13.32 \%$ from $3.83 \%$ in control group after 24 h hypoxia. It was reduced to $3.43 \%$ in tanshinone IIA treatment group.

Tanshinone IIA upregulates miR-133 expression in hypoxic cells

The level of miR-133 was detected by real-time RT-PCR assay. The expression of miR-133 was increased in hypoxic cardiomyocytes ( 2.48 fold vs control). But it was further elevated in hypoxic cardiomyocytes treated with tanshinone IIA as shown in Fig. 3 (3.25 fold vs control, $\mathrm{p}<0.05$ ).

\section{Tanshinone IIA activates MAPK ERK1/2 signaling}

To shed some lights on the potential mechanisms underlying the cytoprotective effects, the effect of tanshinone IIA on MAPK signaling pathway was measured on hypoxic cardiomyocytes. Under hypoxic condition, MAPK ERK1/2 was activated in cultured cardiomyocytes. Interestingly, MAPK ERK1/2 was activated further in cells treated with tanshinone IIA compared with hypoxic cells (Fig. 4). 
Fig. 5. The specific ERK1/2 Inhibitor, U0126, protects NRVCs against hypoxic injury. U0126 was pre-incubated for $30 \mathrm{~min}$. After exposure of NRVCs to hypoxic condition for $24 \mathrm{~h}$, miR-133 level (A) and expression of ERK1/2 protein levels (B) were detected respectively. Data are plotted as the mean $\left( \pm\right.$ S.E.M). ${ }^{*} p<0.05$ vs. control; $\# \mathrm{p}<0.05$ vs. hypoxia, $\mathrm{n}=5$.

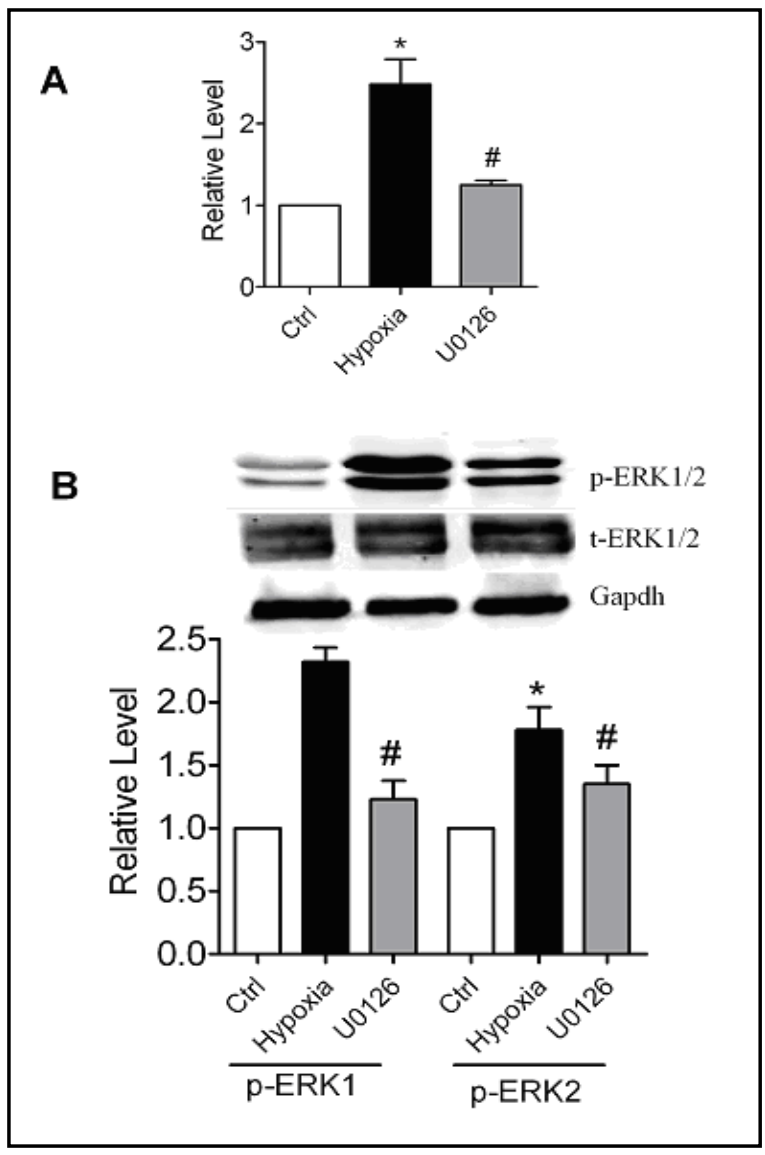

To make sure whether activated ERK1/2 could modulate miR-133, we used U0126, the specific inhibitor of ERK1/2 to detect the change of miR-133 after hypoxia. Compared with hypoxic cells, the level of miR-133 decreased significantly in cells treated with U0126 $(\mathrm{P}<0.05$, Fig. 5A). Meanwhile, U0126 blocked the phosphorylation level of ERK1/2 induced by hypoxia stimulation $(\mathrm{P}<0.05$, Fig. $5 \mathrm{~B})$, which suggested that ERK1/2 may be involved in the regulation of miR-133, which in turn plays a crucial role in protecting cardiomyocytes against hypoxic injury.

\section{Discussion}

Cardiomyocytes apoptosis could be induced by hypoxia, myocardial infarction (MI), ischemia-reperfusion (IR), or $\mathrm{H}_{2} \mathrm{O}_{2}[22,30,31]$. Previous evidence indicated that apoptosis occurred in cardiovascular diseases and may be a risk factor in the development of MI and heart failure [32]. In fact, hypoxia itself was sufficient to induce apoptosis in primary cultured neonatal and adult cardiac myocytes, suggesting that decreased availability of oxygen could be a proximate stimulus for myocyte apoptosis during ischemia [22].

Tanshione IIA is isolated from Traditional Chinese Medicine, Salvia militiorrhiza. Itshowed cardioprotectiveactivity, and has been widelyused in the treatment of cardiovascular diseases, such as myocardial infarction, angina pectoris, et al. [17, 33]. Our previous studies also showed that tanshinone IIA reversed cardiac potassium channel remodeling in postinfarcted heart through depressing SRF-induced miR-1 overexpression [17]. Inhibition of p38 MAPK by tanshinone IIA contributed to the downregulation of elevated expression of miR-1 in ischemic and hypoxic cardiomyocytes [18]. Further study showed that tanshinone IIA attenuated cardiomyocyte hypertrophy induced by isoprenaline through inhibiting 
Calcineurin/NFATc3 pathway [34]. On the other hand, tanshinone IIA was proved to protect brain tissues against ischemic and hypoxic damage in vivo and in vitro by mediating brain iron homeostasis [35]. These studies provide new insights into the pharmacological mechanism of tanshinone IIA in cardiovascular diseases.

In this study, we found that cell viability was markedly reserved with pretreatment of tanshinone IIA compared with that of the hypoxic cardiomyocyte. The expression of miR-133 was increased during hypoxia, suggesting that a self-protection mechanism was turned on when the cells underwent injury. It has been proved that miR-133a was involved in the protection of myocardial ischemia by regulating members of the apoptosis-related caspase-9 family [10]. Our results showed that tanshinone IIA upregulated miR-133 through the MAPK ERK1/2 signaling pathway. When cardiomyocytes in hypoxic environment were pretreated with U0126, no elevation of miR-133 has been observed. In addition, there were no significant difference of the miR-133 level with and without tanshinone IIA treatment under normoxia, so did U0126. Data were not shown.

Many signaling pathways convey apoptotic stimuli in cardiomyocytes [29]. Stress or $\mathrm{H}_{2} \mathrm{O}_{2}$ stimulation activates MAPKs and various intracellular target proteins, leading to cardiomyocytes apoptosis, necrosis, and eventual heart dysfunction. Activation of JNK (cJun $\mathrm{N}$-terminal kinases), or p38 signaling pathway was regarded as a disadvantageous and deleterious indicator in hypoxic cardiomyocytes, but the action of ERK1/2 was thought to be beneficial [36]. JNK and p38 MAPK induce myocardial apoptosis in response to hypoxia stimulation [32, 37]. However, ERK1/2 activation was enhanced in response to ischemia preconditioning, which reduced cell death and resisted insults [38].

Our result showed that the MAPK ERK1/2 was activated by hypoxia and further increased with tanshinone IIA treatment. The activation of ERK1/2 probably upregulates miR-133 by some transcription factors, such as SRF. Accompanied with the higher level of ERK1/2 expression, the level of miR-133 was also further increased in the hypoxic cardiomyocytes with pretreatment of tanshinone IIA. Increased miR-133 may contribute somewhat to antiapoptotic effect of tanshinone IIA, since miR-133 was reported to protect cardiomyocytes against apoptosis by inhibiting caspase-9 [10].

Collectively, our study revealed a novel mechanism about the protective effect of tanshinone IIA on cardiomyocytes. Tanshinone IIA enhanced the resistance to a hypoxic insult through activation of ERK1/2, which subsequently upregulate miR-133 expression in neonatal cardiomyocytes.

\section{Acknowledgements}

This work was supported in part by the Funds for Creative Research Groups (81121003) and the Major Program (81130088) of National Natural Science Foundation of China, and the National Nature Science Foundation of China (No31171094 and 81001434), and New Century Training Programme Foundation for the Talents (1155-NCET-010) of Heilongjiang Province of China to H.S.

\section{References}

1 Jackson RJ, Standart N: How do microRNAs regulate gene expression? Sci STKE 2007;2007:re1.

2 Roy S, Khanna S, Hussain SR, Biswas S, Azad A, Rink C, Gnyawali S, Shilo S, Nuovo GJ, Sen CK: MicroRNA expression in response to murine myocardial infarction: MiR-21 regulates fibroblast metalloprotease-2 via phosphatase and tensin homologue. Cardiovasc Res 2009;82:21-29. 


\begin{tabular}{|c|c|c|}
\hline Cellular P & Cell Physiol Biochem 2012;30:843-852 & \\
\hline and Biochemistry & $\begin{array}{l}\text { DOI: } 10.1159 / 000341462 \\
\text { Published online: August } \\
\text { 10, } 2012\end{array}$ & $\begin{array}{l}\text { O } 2012 \text { S. Karger AG, Basel } \\
\text { www.karger.com/cpb }\end{array}$ \\
\hline
\end{tabular}

-3 van Rooij E, Sutherland LB, Thatcher JE, DiMaio JM, Naseem RH, Marshall WS, Hill JA, Olson EN: Dysregulation of microRNAs after myocardial infarction reveals a role of miR-29 in cardiac fibrosis. Proc Natl Acad Sci USA 2008;105:13027-13032.

4 Shan H, Zhang Y, Lu Y, Pan Z, Cai B, Wang N, Li X, Feng T, Hong Y, Yang B: Downregulation of miR-133 and miR-590 contributes to nicotine-induced atrial remodelling in canines. Cardiovasc Res 2009;83:465-472.

-5 Zhao Y, Samal E, Srivastava D: Serum response factor regulates a muscle-specific microRNA that targets hand2 during cardiogenesis. Nature 2005;436:214-220.

6 Yang B, Lin H, Xiao J, Lu Y, Luo X, Li B, Zhang Y, Xu C, Bai Y, Wang H, Chen G, Wang Z: The muscle-specific microRNA miR-1 regulates cardiac arrhythmogenic potential by targeting GJA1 and KCNJ2. Nat Med 2007;13:486-491.

7 Care A, Catalucci D, Felicetti F, Bonci D, Addario A, Gallo P, Bang ML, Segnalini P, Gu Y, Dalton ND, Elia L, Latronico MV, Hoydal M, Autore C, Russo MA, Dorn GW 2nd, Ellingsen O, Ruiz-Lozano P, Peterson KL, Croce CM, Peschle C, Condorelli G: MicroRNA-133 controls cardiac hypertrophy. Nat Med 2007;13:613-618.

-8 Dong DL, Chen C, Huo R, Wang N, Li Z, Tu YJ, Hu JT, Chu X, Huang W, Yang BF: Reciprocal repression between microRNA-133 and calcineurin regulates cardiac hypertrophy: A novel mechanism for progressive cardiac hypertrophy. Hypertension 2010;55:946-952.

-9 Duisters RF, Tijsen AJ, Schroen B, Leenders JJ, Lentink V, van der Made I, Herias V, van Leeuwen RE, Schellings MW, Barenbrug P, Maessen JG, Heymans S, Pinto YM, Creemers EE: MiR-133 and miR-30 regulate connective tissue growth factor: Implications for a role of microRNAs in myocardial matrix remodeling. Circ Res 2009;104:170-178, 6p following 178.

-10 He B, Xiao J, Ren AJ, Zhang YF, Zhang H, Chen M, Xie B, Gao XG, Wang YW: Role of miR-1 and miR-133a in myocardial ischemic postconditioning. J Biomed Sci 2011;18:22.

-11 Adams JD, Wang R, Yang J, Lien EJ: Preclinical and clinical examinations of salvia miltiorrhiza and its tanshinones in ischemic conditions. Chin Med 2006;23:3.

$\checkmark 12$ Cheng TO: Cardiovascular effects of danshen. Int J Cardiol 2007;121:9-22.

13 Gao J, Yang G, Pi R, Li R, Wang P, Zhang H, Le K, Chen S, Liu P: Tanshinone IIA protects neonatal rat cardiomyocytes from adriamycin-induced apoptosis. Transl Res 2008;151:79-87.

14 Yang L, Zou X, Liang Q Chen H, Feng J, Yan L, Wang Z, Zhou D, Li S, Yao S, Zheng Z: Sodium tanshinone IIA sulfonate depresses angiotensin II-induced cardiomyocyte hypertrophy through MEK/ERK pathway. Exp Mol Med 2007;39:65-73.

15 Yang R, Liu A, Ma X, Li L, Su D, Liu J: Sodium tanshinone IIA sulfonate protects cardiomyocytes against oxidative stress-mediated apoptosis through inhibiting JNK activation. J Cardiovasc Pharmacol 2008;51:396-401.

16 Therapeutic effect of sodium tanshinone IIA sulfonate in patients with coronary heart disease. A double blind study. Shanghai cooperative group for the study of tanshinone IIA.J Tradit Chin Med 1984;4:20-24.

-17 Shan H, Li X, Pan Z, Zhang L, Cai B, Zhang Y, Xu C, Chu W, Qiao G, Li B, Lu Y, Yang B: Tanshinone IIA protects against sudden cardiac death induced by lethal arrhythmias via repression of microRNA-1. Br J Pharmacol 2009;158:1227-1235.

18 Zhang Y, Zhang L, Chu W, Wang B, Zhang J, Zhao M, Li X, Li B, Lu Y, Yang B, Shan H: Tanshinone IIA inhibits miR-1 expression through p38 MAPK signal pathway in post-infarction rat cardiomyocytes. Cell Physiol Biochem 2010;26:991-998.

19 Ravingerova T, Barancik M, Strniskova M: Mitogen-activated protein kinases: A new therapeutic target in cardiac pathology. Mol Cell Biochem 2003;247:127-138.

-20 Kim HA, Rhim T, Lee M: Regulatory systems for hypoxia-inducible gene expression in ischemic heart disease gene therapy. Adv Drug Deliv Rev 2011;63:678-687.

-21 Chu W, Li X, Li C, Wan L, Shi H, Song X, Liu X, Chen X, Zhang C, Shan H, Lu Y, Yang B: TGFBR3, a potential negative regulator of TGF-beta signaling, protects cardiac fibroblasts from hypoxia-induced apoptosis. J Cell Physiol 2011;226:2586-2594.

-22 Tanaka M, Ito H, Adachi S, Akimoto H, Nishikawa T, Kasajima T, Marumo F, Hiroe M: Hypoxia induces apoptosis with enhanced expression of fas antigen messenger RNA in cultured neonatal rat cardiomyocytes. Circ Res 1994;75:426-433.

23 Lips DJ, Bueno OF, Wilkins BJ, Purcell NH, Kaiser RA, Lorenz JN, Voisin L, Saba-El-Leil MK, Meloche S, Pouyssegur J, Pages G, De Windt LJ, Doevendans PA, Molkentin JD: MEK1-ERK2 signaling pathway protects myocardium from ischemic injury in vivo. Circulation 2004;109:1938-1941. 


\section{Cellular Physiology $\quad$ Cell Physiol Biochem 2012;30:843-852 and Biochemistry

-24 Liao Z, Brar BK, Cai Q Stephanou A, O’Leary RM, Pennica D, Yellon DM, Latchman DS: Cardiotrophin-1 (CT-1) can protect the adult heart from injury when added both prior to ischaemia and at reperfusion. Cardiovasc Res 2002;53:902-910.

25 Yue TL, Wang C, Gu JL, Ma XL, Kumar S, Lee JC, Feuerstein GZ, Thomas H, Maleeff B, Ohlstein EH: Inhibition of extracellular signal-regulated kinase enhances ischemia/reoxygenation-induced apoptosis in cultured cardiac myocytes and exaggerates reperfusion injury in isolated perfused heart. Circ Res 2000;86:692-699.

26 Lu Y, Zhang Y, Wang N, Pan Z, Gao X, Zhang F, Shan H, Luo X, Bai Y, Sun L, Song W, Xu C, Wang Z, Yang B: MicroRNA-328 contributes to adverse electrical remodeling in atrial fibrillation. Circulation 2010;122:2378-2387.

-27 Lu Y, Zhang Y, Shan H, Pan Z, Li X, Li B, Xu C, Zhang B, Zhang F, Dong D, Song W, Qiao G, Yang B: MicroRNA-1 downregulation by propranolol in a rat model of myocardial infarction: A new mechanism for ischaemic cardioprotection. Cardiovasc Res 2009;84:434-441.

28 Wang H, Zhang Y, Cao L, Han H, Wang J, Yang B, Nattel S, Wang Z: Herg K+ channel, a regulator of tumor cell apoptosis and proliferation. Cancer Res 2002;62:4843-4848.

-29 Zhang L, Xu CQ, Hong Y, Zhang JL, Liu Y, Zhao M, Cao YX, Lu YJ, Yang BF, Shan HL: Propranolol regulates cardiac transient outward potassium channel in rat myocardium via cAMP/PKA after short-term but not after long-term ischemia. Naunyn Schmiedebergs Arch Pharmacol 2010;382:63-71.

-30 Kajstura J, Cheng W, Reiss K, Clark WA, Sonnenblick EH, Krajewski S, Reed JC, Olivetti G, Anversa P: Apoptotic and necrotic myocyte cell deaths are independent contributing variables of infarct size in rats. Lab Invest 1996;74:86-107.

-31 Gottlieb RA, Burleson KO, Kloner RA, Babior BM, Engler RL: Reperfusion injury induces apoptosis in rabbit cardiomyocytes. J Clin Invest 1994;94:1621-1628.

-32 Haunstetter A, Izumo S: Apoptosis: Basic mechanisms and implications for cardiovascular disease. Circ Res 1998;82:1111-1129.

33 Fu J, Huang H, Liu J, Pi R, Chen J, Liu P: Tanshinone IIA protects cardiac myocytes against oxidative stresstriggered damage and apoptosis. Eur J Pharmacol 2007;568:213-221.

-34 Tan X, Li J, Wang X, Chen N, Cai B, Wang G, Shan H, Dong D, Liu Y, Li X, Yang F, Zhang P, Yang B, Lu Y: Tanshinone IIA protects against cardiac hypertrophy via inhibiting calcineurin/nfatc3 pathway. Int J Biol Sci 2011;7:383-389.

-35 Yang L, Zhang B, Yin L, Cai B, Shan H, Zhang L, Lu Y, Bi Z: Tanshinone IIA prevented brain iron dyshomeostasis in cerebral ischemic rats. Cell Physiol Biochem 2011;27:23-30.

36 Hausenloy DJ, Yellon DM: Survival kinases in ischemic preconditioning and postconditioning. Cardiovasc Res 2006;70:240-253.

-37 Webster KA: Programmed death as a therapeutic target to reduce myocardial infarction. Trends Pharmacol Sci 2007;28:492-499.

38 Krishnamurthy P, Subramanian V, Singh M, Singh K: Beta1 integrins modulate beta-adrenergic receptorstimulated cardiac myocyte apoptosis and myocardial remodeling. Hypertension 2007;49:865-872. 